This is the author's final, peer-reviewed manuscript as accepted for publication. The publisher-formatted version may be available through the publisher's web site or your institution's library.

\title{
Multi-couple group and self-directed PREP formats enhance relationship satisfaction and improve anger management skills in Air Force couples
}

Jared R. Anderson, Sandra M. Stith, Matthew D. Johnson, Marjorie M. StrachmanMiller, Yvonne Amanor-Boadu, David J. Linkh

\section{How to cite this manuscript}

If you make reference to this version of the manuscript, use the following information:

Anderson, J. R., Stith, S. M., Johnson, M. D., Strachman-Miller, M. M., Amanor-Boadu, Y., \& Linkh, D. J. (2013). Multi-couple group and self-directed PREP formats enhance relationship satisfaction and improve anger management skills in Air Force couples. Retrieved from http://krex.ksu.edu

\section{Published Version Information}

Citation: Anderson, J. R., Stith, S. M., Johnson, M. D., Strachman-Miller, M. M., Amanor-Boadu, Y., \& Linkh, D. J. (2013). Multi-couple group and self-directed PREP formats enhance relationship satisfaction and improve anger management skills in Air Force couples. The American Journal of Family Therapy, 41(2), 121-133.

Copyright: Copyright (C) Taylor \& Francis Group, LLC

Digital Object Identifier (DOI): doi:10.1080/01926187.2012.671104

Publisher's Link: http://www.tandfonline.com/doi/abs/10.1080/01926187.2012.671104

This item was retrieved from the K-State Research Exchange (K-REx), the institutional repository of Kansas State University. K-REx is available at http://krex.ksu.edu 
Multi-Couple Group and Self-Directed PREP Formats Enhance Relationship Satisfaction and Improve Anger Management Skills in Air Force Couples

Jared R. Anderson and Sandra M. Stith

Kansas State University

Matthew D. Johnson

University of Alberta

Marjorie M. Strachman-Miller and Yvonne Amanor-Boadu

Kansas State University

David J. Linkh

U.S. Air Force Family Advocacy Program

Jared R. Anderson, Sandra M. Stith, Marjorie M. Strachman-Miller, Yvonne Amanor-Boadu, School of Family Studies and Human Services, Kansas State University; Matthew D. Johnson, Department of Human Ecology, University of Alberta; David J. Linkh, U.S. Air Force Family Advocacy Program

Acknowledgements: The authors would like to thank Col. Fred Stone, Col. Carol Copeland, Major Rachel E. Foster, Major Wendy J. Travis, Bettye Williams and the Air Force Family Advocacy Program for their contributions to this research project.

This material is based upon work supported by the Cooperative State Research, Education, and Extension Service, U.S. Department of Agriculture, and the U.S. Air Force, under Agreement No. 09-EXCA-48353. Any opinions, findings, conclusions, or recommendations expressed in this publication are those of the author(s) and do not necessarily reflect the views of the U.S. Department of Agriculture or the U.S. Air Force.

Correspondence concerning this article should be addressed to Jared R. Anderson, School of Family Studies and Human Services, Kansas State University, 113 Campus Creek Complex, Manhattan, KS 66506. E-mail: jra@ksu.edu. Phone: 785-532-4198. 


\begin{abstract}
This study examined the effectiveness of delivering the Prevention and Relationship Enhancement Program (PREP) to volunteer couples in the U.S. Air Force in either a six-week instructional multi-couple group condition or a self-directed book condition. A standardized pre/post protocol was employed and outcomes included relationship satisfaction and anger management skills, two known risk factors for partner maltreatment. Significant improvement was found for the group condition in both relationship satisfaction and anger management skills, but only for anger management skills in the self-directed book condition. Finally, the group condition reported greater program satisfaction. Implications are discussed.
\end{abstract}

Keywords: Relationship Education, Air Force, Relationship Satisfaction, Anger Management Skills 
Marriage and relationship education (MRE) programs have generally demonstrated effectiveness in improving relationship quality at least in the short-term (Blanchard, Hawkins, Baldwin, \& Fawcett, 2009; Hawkins, Blanchard, Baldwin, \& Fawcett, 2008). Despite these fairly robust results, the literature is limited by the almost sole focus on relationship satisfaction and communication (Hawkins et al., 2008). In addition, traditional delivery formats which typically include multi-couple groups have failed to attract a diverse set of participants. Participants tend to be homogenous with respect to ethnicity, more religious, and less likely to cohabitate before marriage (Halford, O’Donnell, Lizzio, \& Wilson, 2006). As a result, recent initial attempts have been made to deliver MRE programming in self-directed formats, including web-based (Braithwaite \& Fincham, 2007, 2009), workbook-based (L'Abate, 2011) and DVD-based formats (Halford, 2011). Since couples are more likely to seek out books and marital therapy as opposed to MRE workshops when experiencing relationship concerns (Doss, Rhoades, Stanley, \& Markman, 2009), it is important that alternative methods of MRE delivery are made available and empirically tested to increase participation and access to these services. The primary goal of this study was to determine whether two different modes of MRE delivery utilizing the Prevention and Relationship Enhancement Program (PREP), a multi-couple group format and a self-directed format, could enhance the relationship satisfaction and anger management skills of Air Force couples. Both modes of delivery were offered through the USAF Family Advocacy Program (FAP) whose mission is to develop, implement, and evaluate programs and policies to prevent and treat family maltreatment within the AF.

\section{MRE Effectiveness in a Multi-Couple Group \& Self-Directed Formats}

The results of three recently published meta-analyses demonstrated that MRE programs produced moderate to large effect sizes for relationship quality and for communication skills and 
results were generally maintained during short-term follow ups (Fawcett, Hawkins, Blanchard, \& Carroll, 2010). PREP is the MRE program with arguably the most empirical evidence of effectiveness. PREP outcome research demonstrated improved communication and problem solving skills, greater relationship satisfaction, and lower dissolution rates for couples up to four years after participating in PREP in several randomized or quasi-experimental, controlled trials (Hahlweg, Markman, Thurmaier, Engl, \& Eckert, 1998; Markman, Renick, Floyd, Stanley, \& Clements, 1993). In addition, couples taking PREP were also less likely to report physical violence compared to a comparison group that did not enroll in PREP (Markman et al., 1993; Stanley, Markman, St. Peters, \& Leber, 1995). Despite these fairly robust results, there are several limitations to the current body of literature, including a focus on multi-couple groups, well-adjusted pre-marital couples, and a primary focus on relationship satisfaction and communication as outcome variables.

Due to the inability of most MRE programs to attract a wide and diverse audience to traditional multi-couple groups, a growing focus on alternative delivery methods, including workbooks and self-directed web and DVD based programs have emerged. For example, L'Abate $(2004 ; 2007 ; 2011)$ has published many low-cost interactive practice exercises or manuals to be used by clients or prevention participants and their therapists with suggestions for ways individuals and couples can improve their mental health and prevent relationship problems in their own homes. Although research on these programs is limited, the initial results indicate that self-directed MRE programs can be as effective as traditional face-to-face programs. For example, in a study of undergraduate students given ePREP, a computer based version of PREP, participants showed significant decreases in anxiety and depression, lower levels of psychological aggression and physical assault, and greater trust at post-test than participants in 
the control group (Braithwaite \& Fincham, 2007). Participants in the ePREP condition did not differ from controls on either communication or relationship satisfaction in either study. Halford and colleagues have developed a DVD based self-directed MRE program called Couple CARE that includes periodic telephone calls from a psychologist. When compared to wait-list control couples, couples participating in Couple CARE had higher levels of relationship satisfaction and for women, improved relationship self-regulation. No differences were found between participants in Couple CARE and control groups on negative communication or on men's self regulation (Halford, Moore, Wilson, Farrugia, \& Dyer, 2004).

\section{PREP as a Potential Tool to Prevent Spousal Maltreatment in the U.S. Air Force}

Marriage and relationship education programs have been identified as potentially valuable interventions for the primary prevention of family maltreatment (Slep \& Heyman, 2008), through their impact on risk factors for spousal maltreatment (Hawkins, 2011). In a metaanalytic review of risk factors for spouse maltreatment, both low relationship satisfaction and poor anger management skills were associated with increased physical aggression (Stith, Smith, Penn, Ward \& Tritt, 2004). Although anger management skills related to PREP have yet to be empirically examined, PREP has been shown to improve communication skills by decreasing negative communication and increasing positive communication. Also as previously discussed, PREP has been shown to have an impact on the frequency of physically violent behaviors (Markman et al., 1993), with lower levels of pushing, shoving, or slapping for PREP couples up to 12 years post-intervention, compared to a comparison group of couples (Stanley, Markman, St. Peters, \& Leber, 1995). In addition, at 10-month follow up, couples in the ePREP condition evidenced less psychological aggression and physical assault than the participants in the control condition (Braithwaite \& Fincham, 2009). These results point to the possibility of MRE 
programs such as PREP serving as a potential tool for preventing spousal maltreatment through increasing relationship satisfaction and skills such as anger management.

\section{Purpose of the Study}

The purpose of this study was to determine whether two types of PREP delivery methods, the traditional multi-couple group format and a self-directed book version (i.e., 12 Hours to a Great Marriage, Markman, Stanley, Blumberg, Jenkins \& Whitely 2004), could effectively improve Air Force couples' relationship satisfaction and anger management skills, known risk factors for spousal maltreatment. A secondary purpose of this study was to determine the benefits and challenges of facilitating these two PREP formats on Air Force bases. The current study also adds to the small, but growing literature on the effectiveness of self-directed MRE approaches as well as adds to the literature by including anger management skills as an outcome variable of MRE intervention. Finally, given the on-going U.S. involvement in Operation Iraqi and Enduring Freedom, the current rates of partner maltreatment in the U.S. Air Force (Foran, Slep, \& Heyman, 2011), and the potential for military service to increase relationship distress and dissolution (Karney \& Crown, 2007), finding programs that support and enhance military relationships is important. It was expected that couples in both PREP intervention formats would improve their relationship satisfaction and anger management skills from pre-test to post-test.

\section{Method}

\section{Program Delivery Methods}

Twelve Air Force (AF) Bases participated in the study. Outreach Managers (OM), social workers at AF Bases who are responsible for coordinating spousal violence prevention efforts, recruited participants and delivered PREP to them at their individual bases. OMs at eight AF bases received PREP training and offered six two-hour sessions of PREP in a multi-couple group 
format. The remaining four bases distributed the 12-chapter book developed by the authors of PREP, 12 Hours to a Great Marriage, to couples volunteering to participate in a self-directed couples enrichment project. Each chapter takes approximately one hour to read and complete the exercises, for a total of 12 hours. The OMs at these bases were asked to contact couples in the self-directed book condition once a week to provide support. The OMs distributed pre-tests to each member of the couple before the couple participated in the group or self-directed book condition. Once the program was completed, participants took either a paper post-test in the last group session or a web-based survey via a link in an email. The OMs were asked to email participants to request their response to the online survey in an attempt at increasing response rates. When the study was completed, OMs were asked to provide responses to a qualitative questionnaire via email to gain their feedback on the benefits and challenges to the method of PREP they facilitated. This information was gathered to assess adjustments that might make both delivery methods more feasible and accessible in the future.

\section{Measures}

Couple Satisfaction Index (CSI-16). The 16-item version of the couples satisfaction index (CSI-16; Funk \& Rogge, 2007) was used to assess pre- and post-intervention marital satisfaction. The CSI-16 is a measure of relationship satisfaction developed through an itemresponse theory analysis of 180 items commonly used to measure relationship satisfaction. Total scores range from 0 to 81 , with higher scores indicating greater satisfaction. Cronbach's alpha reliability in the current study is .98 at both pre- and post-intervention.

Anger Management Scale (AMS). The 12-item Anger Management Scale (Stith \& Hamby, 2002) assessed pre- and post-intervention anger management skills. The AMS assesses concrete, specific cognitions and behaviors that can increase or decrease anger in intimate 
partner relationships and, therefore, influences the respondent's level of partner violence. Total scores ranged from 12 to 48 with higher scores indicating greater ability to manage anger. Alpha reliability in the current study is .61 at pre-intervention and .60 at post-intervention.

Program Satisfaction. Six questions were used to assess overall satisfaction with the program. Total scores ranged from 6 to 30 with higher scores indicating greater satisfaction with the program. Alpha reliability in the current study is .92 .

Demographics. A number of demographic questions were included in order to define the current sample: rank, military status, age, number of children in the home, whether or not they had a previous maltreatment incident that led to a Family Advocacy Program referral (FAP referral) and deployment history.

\section{Attrition \& Preliminary Analyses}

A significant number of participants in each group did not complete the post-intervention assessment. As discussed previously, OMs who facilitated the multi-couple groups had the option of providing post-test assessments at the close of the final group session or on-line, whereas the self-directed book participants completed the post-test on-line. On-line post-test participation was low. Attrition rates ranged from $42.6 \%$ for the multi-couple group to $54.7 \%$ for the self-directed book condition. To determine whether attrition from the study was random, we conducted a series of binary logistic regression analyses $(0=$ non-completers, $1=$ completers $)$ to determine whether demographic and outcome variables predicted post-test completion. Variables in the attrition analyses included age, gender, rank, FAP referral, number of children in the home, deployment history, and the outcome variables of relationship satisfaction and anger management skills. There were no statistically significant coefficients for any of the variables. Thus, our subsequent analyses include only those participants who completed both pre and post 
intervention assessments. This reduced sample included 107 participants, 78 participants in the multi-couple group format and 29 participants in the self-directed book condition.

Since participants were not randomly assigned to groups, we first examined the sample on the demographic and outcome variables to determine whether differences existed between the multi-couple group participants and the self-directed book participants. For an overview of participant demographic variables see Table 1. A series of independent samples $t$-tests and chisquare analyses revealed significant differences between the participants in the two formats. Specifically, statistically significant differences were found related to age, $t(105)=-2.70, p<$ .01 ; relationship satisfaction pre-intervention, $t(105)=-2.56, p<.05$; anger management skills pre-intervention, $t(104)=-1.92, p<.10 ; \operatorname{rank}, \chi^{2}(1)=9.63, p<.01$; education, $\chi^{2}(3)=10.75, p<$ .05 ; and FAP referral, $\chi^{2}(1)=4.14, p<.05$. There were no significant differences between the groups on the number of children in the home, relationship status, and deployment status within the last year. Participants who completed the self-directed book group were, on average, older $(M=35.41, S D=2.02$ versus $M=29.53, S D=0.82)$, had significantly higher relationship satisfaction scores $(M=59.21, S D=2.77$ versus $M=49.78, S D=2.00)$ prior to receiving the intervention, held a higher rank, had higher levels of education and were less likely to have experienced a FAP referral than participants who completed the multi-couple group format.

\section{Results}

Given the statistically significant differences on a number of outcome and demographic variables between the two groups, direct comparisons related to treatment effectiveness were not warranted. Therefore, the analyses focus on changes in outcomes separately for each treatment condition. To test the effects of each treatment condition on changes in relationship satisfaction and anger management skills scores we performed two way (time x gender) analysis of variance. 
There was a significant main effect for time related to both relationship satisfaction (pretest $M=49.8, \mathrm{SD}=17.6$; post-test $M=53.8, \mathrm{SD}=17.6, F(1,76)=6.91, p<.01$ ), and anger management skills, (pretest $M=32.2, \mathrm{SD}=4.2$; post-test $M=34.6, \mathrm{SD}=4.0, F(1,74)=31.79$, $p<.001)$ for the multi-couple group condition, suggesting that individuals who participated in the multi-couple group reported improved relationship satisfaction and anger management skills post-intervention. There was no significant Time $\mathrm{x}$ Gender interaction for either relationship satisfaction, $F(1,76)=0.013, p>.05$ or anger management skills, $F(1,74)=3.18, p>.05$, suggesting that men and women evidenced similar levels of improvement between pre- and postintervention. There was also a significant main effect for time related to anger management skills, (pretest $M=33.9, \mathrm{SD}=3.3$; post-test $M=35.2, \mathrm{SD}=3.5, F(1,27)=23.85, p<.05)$, for the self-directed book condition, suggesting that individuals who participated in the self-directed group evidenced improved anger management skills. There was no main effect for Time related to relationship satisfaction (pretest $M=59.2, \mathrm{SD}=14.9$; post-test $M=61.5, \mathrm{SD}=14.3$ ) and no Time x Gender interaction for either relationship satisfaction or anger management skills, suggesting that relationship satisfaction did not significantly improve and that men and women evidenced similar levels of improvement on anger management for the self-directed book group. Finally, an independent samples $t$-test examined differences between groups with respect to program satisfaction. On average, though all participants were generally satisfied with their intervention group, participants who enrolled in the multi-couple group were more satisfied $(M=$ 26.21, $S D=0.44$ ) with their experience than participants who enrolled in the self-directed book group $(M=24.16, S D=0.80 ; t(98)=2.29, p<.05)$.

\section{Feedback From Program Leaders}


The OMs who recruited and delivered PREP to participants at the Air Force bases provided qualitative feedback on the barriers, challenges, and benefits of the delivery method of PREP that they used. Some benefits for couples that the social workers saw for the self-directed book condition included privacy, convenience, and more time to discuss the materials. For example, one OM who delived the self-directed method said, "I think the clients liked it that I wasn't greatly involved in their discussions." Some challenges of the self-directed book method were recruitment, the time it took for them to make individualized weekly calls, participant follow-through on reading and program compliance. OMs who conducted the PREP multicouple groups found benefits in the support and feedback that couples received and participant commitment to the program within a structured group. Facilitators also found that they were able to receive immediate feedback from the group, assess for risk factors, and save time by delivering the program to several couples at once. Challenges of the group included inconsistent attendance, coordinating meeting times, higher cost for materials and training, and less time for discussion. Drop-outs were a problem for many OMs, such as one who stated, "Even couples who initially seemed quite invested in the group process sometimes ended up dropping out."

The OMs were also asked for advice for delivering services in a self-directed or group setting. Group facilitators recommended flexibility when scheduling, keeping the group short, and holding make-up classes. They also offered ideas to make the classes more interesting and accessible, such as providing food and childcare when funding is possible. OMs who faciliated the group also reported using creativity in engaging couples, such as "clever" marketing campaigns and providing weekly supportive emails to couples. Several OMs also suggested providing both conditions and allowing couples to take advantage of the option best for them.

\section{Discussion}


The purpose of the current study was to determine whether two types of PREP delivery methods, the traditional multi-couple group format and a self-directed book version, could improve Air Force couples' relationship satisfaction and anger management skills. The results largely conformed to our expectations. Both conditions were associated with improvement in anger management skills and relationship satisfaction, albeit the improvement in relationship satisfaction was not statistically significant for the self-directed book group. Several factors could explain why the self-directed book group did not evidence statistically significant change in relationship satisfaction scores. First, the vast majority of individuals $(78.3 \%)$ in this group were in the non-distressed range on relationship satisfaction initially. A "ceiling effect" has been discussed in evaluating the effectiveness of MREs because often the participants are already highly satisfied with their relationship prior to the intervention (Fawcett et al., 2010). This could very well be the case for many participants in the self-directed book group, given their significantly higher level of marital satisfaction prior to receiving the intervention. Therefore, it is more likely that couples saw this as an enrichment opportunity to enhance and maintain an already strong relationship rather than to turn around a struggling relationship. Second, the small sample size likely reduced the power of the statistical tests to detect significant changes in scores. Finally, it also could be that the treatment itself was not powerful enough to effect relationship satisfaction scores. These results are similar to those found for the ePREP program and Couple CARE (at least for men), where relationship satisfaction scores were not significantly different from control group relationship satisfaction scores post-intervention (Braithwaite \& Fincham, 2000; Halford et al., 2004). Clearly, additional research is necessary to determine the effectiveness of self-directed approaches in influencing relationship satisfaction and what factors, such as treatment adherence and dosage, contribute to changes. 
While PREP has been associated with lower levels of partner violence in previous research (Braithwaite \& Fincham, 2009; Markman et al., 1993), this study points to the potential mechanism responsible for the reduction in violence: increased anger management skills. It is possible that through learning more effective communication and conflict resolution skills, participants in PREP increase the ability to control their anger when disagreeing with their partner. Longitudinal research exploring the effectiveness of MRE would undoubtedly benefit from examining the interplay between increased anger management skills and partner violence.

The significant pre-intervention differences noted between treatment groups on a number of demographic variables and on relationship satisfaction were an interesting finding. Research has shown that different recruitment methods often capture participants with different backgrounds and more or less diverse samples (Karney, Davila, Cohan, Sullivan, Johnson, \& Bradbury, 1995). It may also be that different MRE formats are more or less appealing to different populations of couples. For example, in the current study, volunteer participants in the self-directed book group were more likely to be officers and to have higher initial relationship satisfaction than were volunteer participants in the multi-couple group format. One explanation for this significant difference in participant rank could be that participant's holding a higher rank may not have wanted to engage in discussions about their marital relationship in the presence of lower-ranked participants. Thus, the self-directed book condition would be more appealing due to the privacy it would provide. On the other hand, selection into the self-directed book condition might have been in response to the couple's deciding that a less-intensive form of treatment was adequate for their situation. Using the CSI-16 cut-off score of 51.5 (Funk \& Rogge, 2007), $21.7 \%$ of participants in the self-directed book condition could be categorized as "distressed" at pre-intervention assessment compared to $46.2 \%$ of participants in the multi-couple group format. 
Likely, these two hypotheses are related. Holding a higher rank would be associated with higher levels of education and higher salaries, two demographic factors closely linked with marital quality (Karney \& Crown, 2007). Therefore, holding a higher rank might increase the likelihood that the couple would have a higher quality marriage (needing less intensive intervention) and would also cause them to avoid participating in treatment with their lower-ranked counterparts (wanting less intensive intervention). Finally, it could also be that those of higher rank (i.e., officers) have more demanding schedules and work related commitments that would preclude them from being able to commit to a weekly group meeting.

While participants in both treatment conditions reported a high level of satisfaction with the program, those in the multi-couple group were significantly more satisfied with the intervention than those in the self-directed book condition. It is possible that those in the multicouple group were presented with additional opportunities from which to derive satisfaction with the intervention. Perhaps the experience was more enjoyable after developing an ongoing relationship with the program facilitator and the other participant couples. This camaraderie would not be possible in a self-directed format. Since program satisfaction was still relatively high for the self-directed book condition, it may not be worthwhile to invest resources in increasing program satisfaction. However, perhaps the type and amount of individual consultation with a facilitator to discuss the material could be a factor that moderates the level of program satisfaction, a hypothesis that could be tested in subsequent studies.

Finally, narrative comments provided by the intervention facilitators highlighted couple retention as a major challenge to both interventions. For the multi-couple group, facilitators reinforced the importance of flexibility in scheduling, offering child care, between session follow-up to keep couples engaged, and utilizing effective marketing strategies. Keeping couples 
engaged in the self-directed book group was also a challenge, as the facilitators reported that many of the couples would fall behind in the reading schedule. Finding ways to maintain regular, effective individual consultation with a facilitator might boost treatment adherence and retention and could also offer an opportunity devise a plan to get couples back on track that might not be following through with the intervention.

\section{Limitations}

The contribution of the current study must be viewed in light of its limitations. First, the absence of random assignment to an intervention group prevented comparisons between the multi-couple and self-directed groups. Future studies might want to compare the effectiveness of different delivery methods for MRE with randomly assigned military couples, something we were unable to do. Second, participant retention was an issue in the current study, with a large portion of couples not completing the intervention, though our attrition rates are similar to other studies in the literature (Stanley et al., 2004). Finally, the current study was comprised of couples who self-selected to participate and cannot be assumed to be representative of all AF couples.

\section{Conclusion}

The current study contributes to the growing literature on the efficacy of alternative treatment delivery methods for MRE (Braithwaite \& Fincham, 2007; Halford et al., 2004) by demonstrating that delivering PREP to Air Force couples in a multi-couple group and selfdirected book format both resulted in increases in anger management and relationship satisfaction from pre- to post-test, although the increase in relationship satisfaction for the selfdirected book condition did not reach statistical significance. These results point to the salience of marriage and relationship education programs to strengthen marriages for Air Force couples. 


\section{References}

Blanchard, V. L., Hawkins, A. J., Baldwin, S. A., \& Fawcett, E. B. (2009). Investigating the effects of marriage and relationship eduation on couples' communication skills: A metaanalytic study. Journal of Family Psychology, 23, 203-214.

Braithwaite, S. R., \& Fincham, F. D. (2007). ePREP: Computer based prevention of relationship dysfunction, depression, and anxiety. Journal of Social and Clinical Psychology, 26, 609622.

Braithwaite, S. R., \& Fincham, F. D. (2009). A randomized clinical trial of a computer based preventive intervention: Replication and extension of ePREP. Journal of Family Psychology, 23, 32-38.

Doss, B. D., Rhoades, G. K., Stanley, S. M., \& Markman, H. J. (2009). Marital therapy, retreats, and books: The who, what, when, and why of relationship help-seeking. Journal of Marital and Family Therapy, 35, 18-29.

Fawcett, E. B., Hawkins, A. J., Blanchard, V. L., \& Carroll, J. S. (2010). Do premarital education programs really work? A meta-analytic study. Family Relations, 59, 232-239.

Foran, H. M., Slep, A. M. S., \& Heyman, R. E. (2011). Prevalences of intimate partner violence in a representative U.S. Air Force sample. Journal of Consulting and Clinical Psychology, 79, 391-397.

Funk, J. L., \& Rogge, R. D. (2007). Testing the ruler with item response theory: Increasing precision of measurement for relationship satisfaction with the couples satisfaction index. Journal of Family Psychology, 21, 572-583. 
Halford, W. K., Moore, E., Wilson, K. L., Farrugia, C., \& Dyer, C. (2004). Benefits of flexible delivery relationship education: An evaluation of the Couple CARE program. Family Relations, 53, 469-476.

Halford, W. K., Moore, E., Wilson, K. L., Farrugia, C., \& Judge, K. (2004). Can couples benefit from a flexible delivery relationship education program. Family Relations, 53, 469-476.

Halford, W. K., O’Donnell, C., Lizzio, A., \& Wilson, K. L. (2006). Do couples at high risk of relationship problems attend premarriage education? Journal of Family Psychology, 20, $160-163$.

Hahlweg, K., Markman, H. J., Thurmaier, F., Engl, J., \& Eckert, V. (1998). Prevention of marital distress: Results of a German prospective longitudinal study. Journal of Family Psychology, 12, 543-556.

Hawkins, A. J. (2011). Programs to help prevent intimate partner violence: Introduction to a special issue. Journal of Couple \& Relationship Therapy, 10, 95-96.

Hawkins, A. J., Blanchard, V. L., Baldwin, S. A., \& Fawcett, E. B. (2008). Does marriage and relationship education work? A meta-analytic study. Journal of Consulting and Clinical Psychology, 76, 723-734.

Karney, B. R., \& Crown, J. S. (2007). Families under stress: An assessment of data, theory, and research on marriage and divorce in the military. Arlington, VA: Rand.

Karney, B. R., Davila, J., Cohan, C. L., Sullivan, K. T., Johnson, M. D., \& Bradbury, T. N. (1995). An empirical investigation of sampling strategies in marital research. Journal of Marriage and the Family, 57, 909-920.

L'Abate, L. (2011). Sourcebook of interactive practice exercises in mental health, Springer Sciences, New York, NY. 
L'Abate, L. (Ed.). (2004). Using workbooks in mental health: Resource in prevention, psychotherapy, and rehabilitation for clinicians and researchers. Binghamton, NY: Haworth.

L'Abate, L. (Ed. ( 2007). Low-cost approaches to promote physical and mental health. New York: Springer Sciences.

Markman, H.J., Renick, M.J., Floyd, F., Stanley, S., \& Clements, M. (1993). Preventing marital distress through communication and conflict management training: A four and five year follow-up. Journal of Consulting and Clinical Psychology, 62, 70-77.

Markman, H.J., Stanley, S.M., Blumberg, S.L., Jenkins, N.H., Whitely, C. (2004). 12 Hours to a Great Marriage; A Step-by-Step Guide for Making Love Last. Jossey-Bass, San Francisco, CA.

Slep, A.M., \& Heyman, R.E. (2008). Public health approaches to family maltreatment prevention: Resetting family psychology's sights from the home to the community. Journal of Family Psychology, 22, 3, 518-528.

Stanley, S. M., Markman, H. J., St. Peters, M., \& Leber, B. D. (1995). Strengthening marriages and preventing divorce: New directions in prevention research. Family Relations, 44, 392.

Stith, S. M., \& Hamby, S. L. (2002). The anger management scale: Development and preliminary psychometric properties. Violence \& Victims, 17, 383-402.

Stith, S. M., Smith, D. B., Penn, C. E., Ward, D. B., \& Tritt, D. (2004). Intimate partner physical abuse prevention and victimization risk factors: A meta-analytic review. Aggression and Violent Behavior, 10, 65-98. 
Table 1

Demographic Data Related to Multi-Couple Group and Self-Directed Book Participants

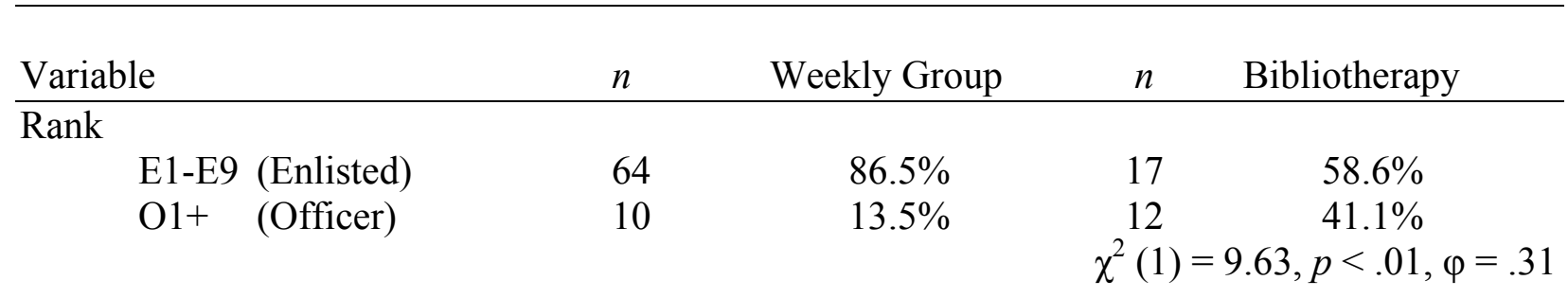

Relationship Status

$\begin{array}{lrrcc}\text { Committed Rel/Engaged } & 8 & 10.3 \% & 7 & 24.1 \% \\ \text { Married } & 70 & 89.7 \% & 22 & 75.9 \% \\ & & & \chi^{2}(1)=3.38, p<.066, \varphi=.18\end{array}$

Highest Level of Education

High School Diploma/Less

Some College 42

$17.9 \%$

$10.3 \%$

College Degree

16

$53.8 \%$

$34.5 \%$

Some Grad/Grad Degree

$20.5 \%$

$24.1 \%$

$7.7 \%$

$31.0 \%$

$\chi^{2}(3)=10.75, p<.05, \varphi=.32$

Family Ever Referred to FAP

Yes

15

$19.2 \%$

$4.3 \%$

63

$80.8 \%$

$96.6 \%$

$\chi^{2}(1)=4.14, p<.05, \varphi=.20$

Deployment within Last Year

Self/Partner/Both

23

Neither

54

$29.9 \%$

$70.1 \%$
6
23
$20.7 \%$
$\chi^{2}(1)=0.89, p=\mathrm{NS}, \varphi=.09$

Age

78

$M=29.5$

$29 \quad M=35.4$

$t(105)=-2.70, p<.01, r=.26$

\# of Children in the Home

78

$M=1.18$

$29 \quad M=1.28$

$t(105)=-0.40, p=\mathrm{NS}, r=.04$ 